\title{
Cassini Dust Measurements at Enceladus and Implications for the Origin of the E ring
}

Frank Spahn ${ }^{1 *}$, Jürgen Schmidt ${ }^{1}$, Nicole Albers ${ }^{1}$, Marcel Hörning ${ }^{1}$, Martin Makuch ${ }^{1}$, Martin Seiß ${ }^{1}$, Sascha Kempf ${ }^{2}$, Ralf Srama ${ }^{2}$, Valeri Dikarev ${ }^{2,3}$, Stefan Helfert ${ }^{2}$, Georg Moragas-Klostermeyer ${ }^{2}$, Alexander V. Krivov ${ }^{3}$, Miodrag Sremčević ${ }^{5}$, Anthony J. Tuzzolino ${ }^{6}$, Thanasis Economou ${ }^{6}$, Eberhard Grün ${ }^{2,4}$

${ }^{1}$ Institut für Physik, Universität Potsdam, Am Neuen Palais 10, Haus 19, D-14469 Potsdam

${ }^{2}$ Max-Planck-Institut für Kernphysik, Heidelberg, Germany

${ }^{3}$ Astrophyskalisches Institut, Friedrich Schiller Universität Jena, Germany ${ }^{4}$ Hawaii Institute of Geophysics and Planetology, University of Hawaii, USA

${ }^{5}$ LASP, University of Colorado, Boulder, USA

${ }^{6}$ LASR, University of Chicago, USA

*Corresponding author: fspahn@agnld.uni-potsdam.de 
During Cassini's close flyby of Enceladus on July 14th 2005 the High Rate Detector of the Cosmic Dust Analyzer registered micron-sized dust particles enveloping this satellite. The dust impact rate peaked about one minute before the closest approach of the spacecraft to the moon. This asymmetric signature is consistent with a locally enhanced dust production in the south polar region of Enceladus. Other Cassini experiments revealed evidence for geophysical activities near Enceladus' south pole: a high surface temperature and a release of water gas. Production or release of dust particles related to these processes may provide the dominant source of Saturn's E ring.

The tenuous E ring is the outermost and largest ring in the Saturnian system, consisting of particles with a peak size between 0.3 and 3 microns (1). The highest density of the E ring and its smallest vertical extent are both observed close to the orbit of Enceladus $(1,2)$, which favors this moon as the main source of that faint ring. In-situ dust measurements at Enceladus with the dust detector aboard the Cassini spacecraft offer the unique opportunity to learn about this satellite, dust production processes at its surface, and ultimately, to shed light on the origin of the E ring.

We report on measurements carried out with the High Rate Detector (HRD) of the Cosmic Dust Analyzer (CDA) during the flyby of Enceladus on July 14, 2005. The detector consists of two thin $(28 \mu \mathrm{m}$ and $6 \mu \mathrm{m})$ polyvenylidene fluoride sensors with cross sections of $50 \mathrm{~cm}^{2}$ and $10 \mathrm{~cm}^{2}(3)$. In this paper we focus on the data collected by the $50 \mathrm{~cm}^{2}$ sensor, which is sensitive for particles with a radius larger than $2 \mu \mathrm{m}$. An impacting hypervelocity grain changes the polarization in the sensor volume resulting in a short, sharp pulse enabling the detector to register up to $10^{4}$ dust impacts per second (4). 
During the flyby a significant increase in the count rate of dust particles was recorded, about 10 minutes before to 10 minutes after closest approach of the spacecraft to the moon (Fig. 1). The peak count rate was 4 particles per second at one minute before closest approach. Similarly, the Cassini ion and neutral mass spectrometer (5) detected water gas, also at a peak rate before closest approach, albeit with a somewhat smaller offset of 30 seconds. This gas plume was also seen by the ultraviolet imaging spectrometer (6) and indirectly, already at an earlier flyby, by the magnetometer ( 7$)$. The time difference between the rate peaks points to a decoupling of gas and dust shortly after both components are released from the satellite surface (8). A gas and dust source near the south pole is compatible with these premature maxima of the rates since the spacecraft approached the moon from south and came closest to Enceladus at a latitude $\approx 25^{\circ} \mathrm{S}$ (Figs. 1 of $(5,9)$ ). In contrast, a dust cloud generated by micrometeoroid impacts, as was observed by the Galileo mission around the Jovian moons (10), would lead to a peak rate directly at closest approach.

The observed particle count rate constrains the production rate of particles at Enceladus while the time offset of its maximum from closest approach can be used to locate those regions on the moon's surface from which the particles originate. Comparing the data to theoretical models of dust production we can estimate the relative contributions of alternative mechanisms of particle creation, yielding implications for the dominant source of the E ring particles. Besides geophysical processes $(11,12)$ micrometeoroid bombardment has been proposed as a particle creation process (13). Two families of micrometeoroids are relevant at Enceladus, namely E ring particles and interplanetary dust particles. The typically large velocities of such projectiles relative to the moon, a few to tens of kilometers per second, make the impacts energetic enough to abundantly produce ejecta at the moon's surface and create a dust cloud $(10,14)$. However, the detection of an 
anomalously high temperature (9) in the south polar region of Enceladus near elongated cracked fractures (15) (dubbed "tiger stripes") lends new support to the idea of E ring dust particles created by cryo-volcanism (ice-volcanoes).

We have modeled the distributions of dust in the vicinity of Enceladus for cases of isotropic ejection of grains from the entire surface and for a localized dust source at the south pole of the moon (Fig. 1). These two cases correspond to the particle production by the impactor-ejecta mechanism and by geological processes at the south pole, respectively. Since the spacecraft's trajectory near closest approach (170 km above surface) lies well inside the Hill sphere (17) of gravitational influence of the moon $\left(r_{h} \approx 948 \mathrm{~km}\right.$, compared to a radius of $252 \mathrm{~km}(5)$ ), an analytical model for the dust cloud developed in (18), based on the two-body approximation, should give an adequate estimate (19). However, to account for the full three-body dynamics near the hill scale, we have numerically simulated the dust configuration around the satellite for both source models. In the simulations particle paths are integrated subject to Saturn's and Enceladus' gravity $(19,20)$. In both simulations 1,000,000 particles are launched from the satellite's surface with starting conditions that are plausible for particles created in an impactor-ejecta process $(16,21)$. To simulate the impactor-ejecta source the starting positions are chosen uniformly over the entire surface of the moon. The localized source is simulated with starting positions distributed uniformly in a circular area of an angular diameter of 30 degrees centered at the south pole, which is on the order of the size of the hot region $(9)$. The motion of the particles governed by the gravity of the planet and the satellite does not depend on the particles' mass or radius, and thus, grains with different radii need not to be distinguished in the simulations. Therefore the size distribution in the model dust cloud near the moon derives from the particle size distribution assumed for the particle creation process.

In the simulations the impactor-ejecta process is found to generate a highly symmetric 
dust configuration in the vicinity of the satellite, as expected, so that the HRD on a flyby through this cloud would observe a maximal count rate directly at closest approach (Fig. 1A). In contrast, the simulated dust ejection from the south pole source reproduces well the observed maximal count rate one minute before closest approach. Fitting combinations of both contributions with a consistent E ring particle background to the data, and requiring that no second peak develops in the rate at closest approach, we can estimate the maximal strength of the impactor-ejecta dust creation at Enceladus relative to that of the south pole source (Fig. 1B). From this fit (HRD data for $R_{p}>2 \mu \mathrm{m}$ ) we can infer the rate of particles larger than 2 micron emitted by the south pole source and escaping the moon's gravity to amount to $5 \times 10^{12}$ particles per second, whereas the impactorejecta mechanism would produce at most $10^{12}$ such particles per second. These numbers correspond to an escaping mass of at least 0.2 kilogram per second, assuming $R_{p}=2 \mu \mathrm{m}$ for all grains. For an extended size distribution this rate may extend to kilograms per second. The E ring particle background, which is naturally contained in the HRD data, has been simulated, following the motion of a particles subject to gravity and perturbation forces $(19,20)$, until they are lost in collisions with Enceladus, other E ring moons, or the main rings. A self-consistent combination of the simulated dust populations is in reasonable agreement with the observed $\mathrm{HRD}$ rate (Fig. 1B).

A differential particle size distribution inferred from the data of both HRD sensors fits to a power law $n\left(R_{p}\right) \propto R_{p}^{\alpha}$ with a slope $\alpha \approx-3$ that remains approximately constant during the flyby (Fig. 1C). This near constance of the exponent indicates that the dynamics of larger grains is dominated by gravity. Such a power law is expected for an impactor-ejecta particle formation scenario (16). On the other hand we showed that the south pole source should be the dominant source of particles. A possible explanation would be that the particles are formed in meteoroid impacts and lifted by south polar gas 
venting. However, an effective acceleration of grains in the gas plume seems implausible for the gas densities inferred from UVIS $(6,15)$.

A side view of the dust configuration from the simulation of the south pole source is shown in Fig. 2, where the absolute numbers are fixed by HRD data at closest approach. A similarly strong stratification of the dust density is evident in images of the dust plume (15). In the simulation the stratification results basically from the power law distribution of particle starting velocities (21).

To investigate the influence of the particle source location on the rate profile measured by HRD, we performed a series of about 2600 simulations, where the source position was systematically varied over the moon's surface. Here, we used 50,000 particles per simulation using for simplicity the initial conditions for the impactor ejecta mechanism (16). For each source we determined the time offset of the peak count rate to closest approach for this flyby. In this way we obtained a contour map of offset times over the moon's surface, which is plotted over an ISS basemap (15) of the geologically active south pole region in Fig. 2. It is found that only a small part of the total surface of Enceladus can have sources which would match the actually observed offset of minus one minute. Interestingly, the region of the "tiger stripes" (covering latitudes $>70^{\circ} \mathrm{S}$ ), is indeed compatible with the data, yielding offsets from -50 to -70 seconds.

Based on simulations of the dust environment around Enceladus we conclude that the Cassini CDA data of the Enceladus flyby on July 14th are compatible with a dust source in the south polar region of the moon. A particle ejection mechanism caused by hypervelocity micrometeoroid impacts alone cannot explain the data. New in-situ measurements of Enceladus' dust cloud will be obtained during a flyby in 2008 at an altitude of only $100 \mathrm{~km}$ over $69^{\circ}$ north. 


\section{References and Notes}

1. P. D. Nicholson, et al., Science 272, 509 (1996).

2. M. R. Showalter, J. N. Cuzzi, S. M. Larson, Icarus 94, 451 (1991).

3. R. Srama et al., Space Science Reviews 114, 465 (2004).

4. This property of the HRD becomes crucial in regions of high particle density - parts of the E ring and in the vicinity of its source satellites - where the impact ionization detector of the CDA is saturated.

5. H. Waite et al. this issue .

6. C. Hansen et al. this issue.

7. M. K. Dougherty et al. this issue.

8. Early decoupling of gas and dust is in accordance with the reported low gas densities (Knudsen regime, (5)) found in the gas plume (6) making molecule-dust collisions unlikely during the time (tens of minutes) from ejection to the impact of the grain at the HRD .

9. J. R. Spencer et al. this issue.

10. H. Krüger, A. V. Krivov, D. P. Hamilton, E. Grün, Nature 399, 558 (1999).

11. P. K. Haff, G. L. Siscoe, A. Eviatar, Icarus 56, 426 (1983).

12. J. S. Kargel, S. Pozio, Icarus 119, 385 (1996).

13. J. E. Colwell, Icarus 106, 536 (1993). 
14. M. Sremčević, A V.Krivov, H. Krüger, F. Spahn, Planet. Space Sci. 53, 625 (2005).

15. C. Porco et al. this issue.

16. A. V. Krivov, M. Sremčević, F. Spahn, V. V. Dikarev, K. V. Kholshevnikov, Planet. Space Sci. 51, 251 (2003).

17. Within the Hill sphere the satellite's gravity is dominating Saturn's gravity. The Hill radius is defined in terms of the masses of Saturn $M_{S}$, of Enceladus $M_{E}$ and distance $a_{E}$ between them as $r_{h}=a_{E} \sqrt[3]{M_{E} / 3\left(M_{S}+M_{E}\right)}$.

18. M. Sremčević, A. V. Krivov, F. Spahn, Planet. Space Sci. 51, 455 (2003).

19. Supplementary material is available on Science Online.

20. Perturbation forces - Saturn's oblateness, Lorentz force, radiative forces - are crucial for the long-term dynamics of the particle and the configuration of the E ring (22-26). They can be neglected for the simulation of dust in the vicinity of Enceladus.

21. Initial velocities follow a power law $N(>v) \propto v^{-2}, 0.15 v_{\text {esc }}<v<2 v_{\text {esc }}$, where $v_{\text {esc }} \approx$ $239 \mathrm{~m} / \mathrm{s}$ is Enceladus' escape velocity. Starting directions are uniformly distributed in a cone of semi-opening angle of $25^{\circ}$ normal to the surface at the starting location.

22. M. Horányi, J. A. Burns, D. P. Hamilton, Icarus 97, 248 (1992).

23. D. P. Hamilton, J. A. Burns, Science 264, 550 (1994).

24. V. V. Dikarev, Astron. Astrophys. 346, 1011 (1999).

25. F. Spahn, K.-U. Thiessenhusen, J. Colwell, R. Srama, E. Grün, J. Geophys. Res. 104, 24111 (1999). 
26. A. Juhász, M. Horányi, Geophys. Res. Lett. 31, 19703 (2004).

27. We thank Torrence V. Johnson for comments on the manuscript. The Potsdam group was supported by the DFG grants Sp 384/16, Sp 384/17, Sp 384/18, DLR grant 50OH0003 and the "Studienstiftung des deutschen Volkes". The group at the MPI für Kernphysik was supported by Max Planck society and by DLR under the grant 500OH9802. The work in Chicago was supported by JPL contract number 961170 and M. Sremčević is funded by the Cassini UVIS project. 
Figure 1: Comparison of the CDA data to simulations. Panel A: Particle density in the plane of Cassini's trajectory estimated from simulations, darker shades referring to a higher density. The impactor-ejecta process (left) leads to a more symmetric dust cloud, while a localized source at the south pole of the moon (right) shows a strong asymmetry. Circles denote the intersection of the plane with the Hill sphere of gravitational influence and the normal projection of the moon's center (not in that plane) is marked by a cross symbol. Cassini's trajectory is plotted as a dash-dotted or dashed line, respectively. The central plot shows the count rates predicted by the simulations computed along the actual spacecraft trajectory, both normalized to the peak rate of the data shown in histogram mode. Panel B: The sum of the rates derived from the two simulations normalized to the observed peak rate together with the simulated E ring background. The maximal strength of the impactor-ejecta process relative to that of the south pole source is chosen in a way that no secondary peak develops in the combined rate near closest approach. Panel $C$ : The slopes of the differential size-distribution $n\left(R_{p}\right) \propto R_{p}^{\alpha}$ versus time to closest approach. The increase of $\alpha$ for $t>4$ minutes is due to a maneuver of Cassini and the related change of the instrument's boresight.

Figure 2: Side view of a simulated dust plume at Enceladus' south pole. Contours of equal column particle density are shown in a Cartesian frame fixed at the center of the moon. The brightest contour denotes $10^{7}$ particles per $m^{2}$, the column density dropping by one half from level to level.

Figure 3: Results of a series of simulations where the position of the source is varied systematically over the moons surface. For each simulation the particle count rate along the Cassini trajectory is computed. The contours of equal time offsets of the maximal 
count rate from closest approach are plotted over a basemap of Enceladus' south pole (15). The thick red line marks the contour of a -60 second offset of the maximal count rate, which was about the value observed by the CDA. Other contours correspond to offsets of $-90,-70,-50,-30$, and zero seconds. The white line around the pole denotes the 77.5 K isotherm from CIRS (9). Cassini's ground track is shown in yellow and the times of rate maxima of the CDA (-50 seconds) and INMS (-30 seconds) (5), as well as the closest approach (CA), are marked. 
A)

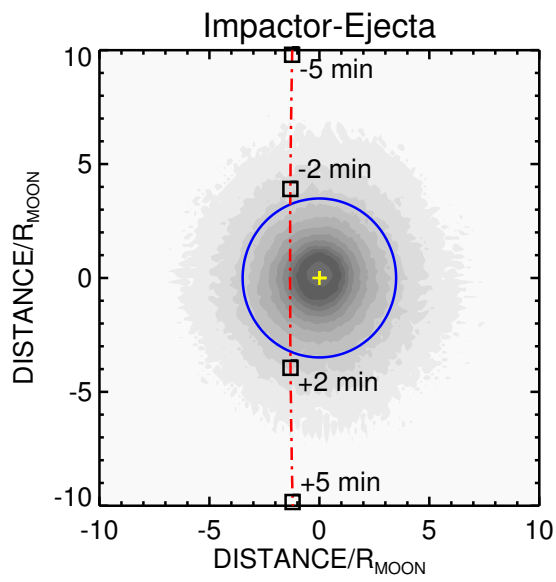

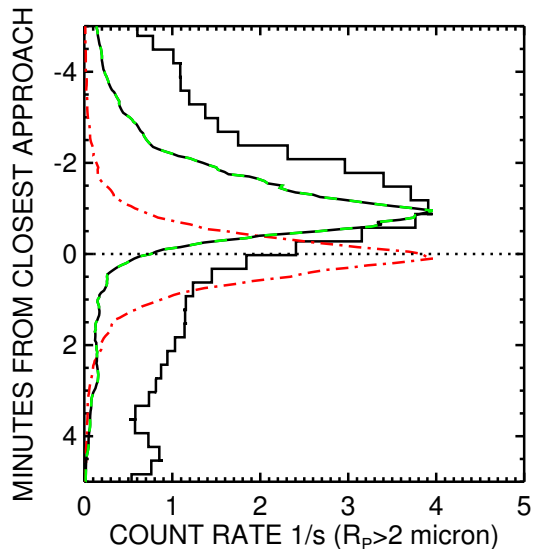

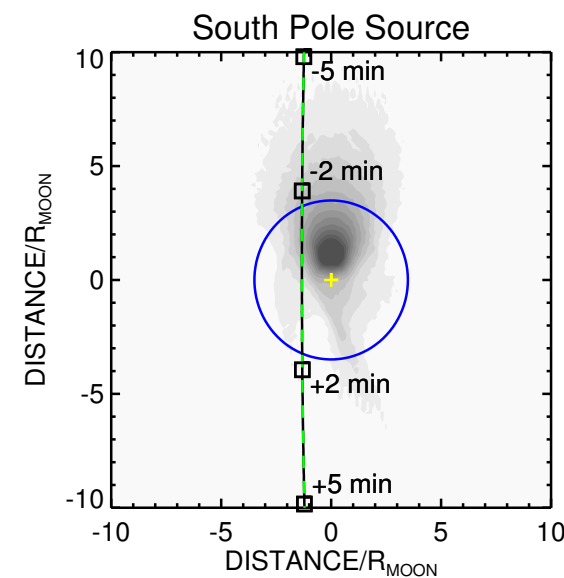

B)

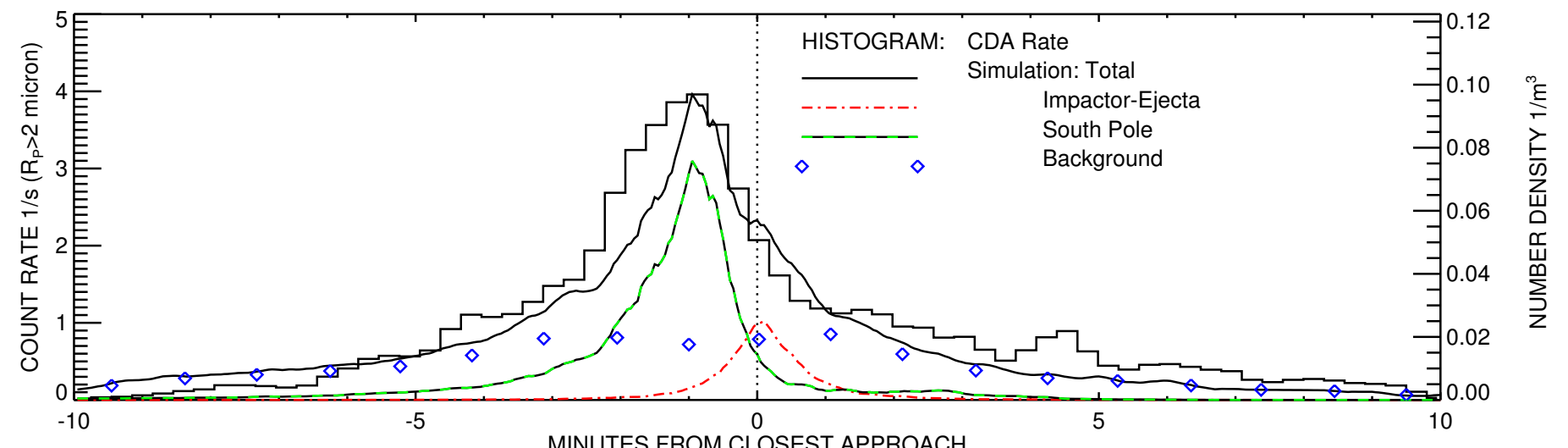

C)

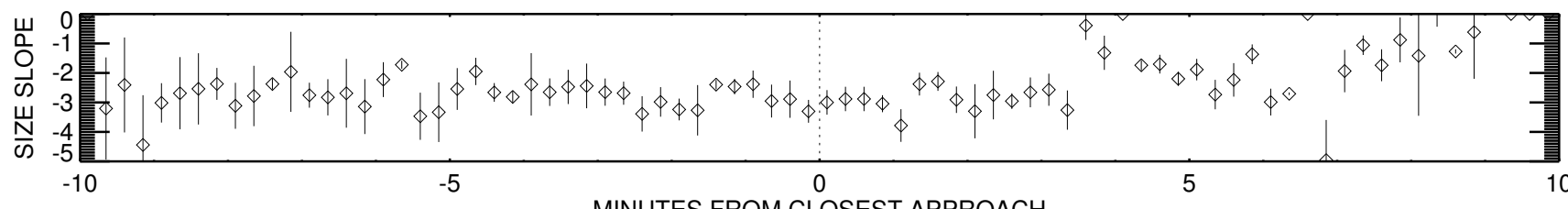

MINUTES FROM CLOSEST APPROACH 


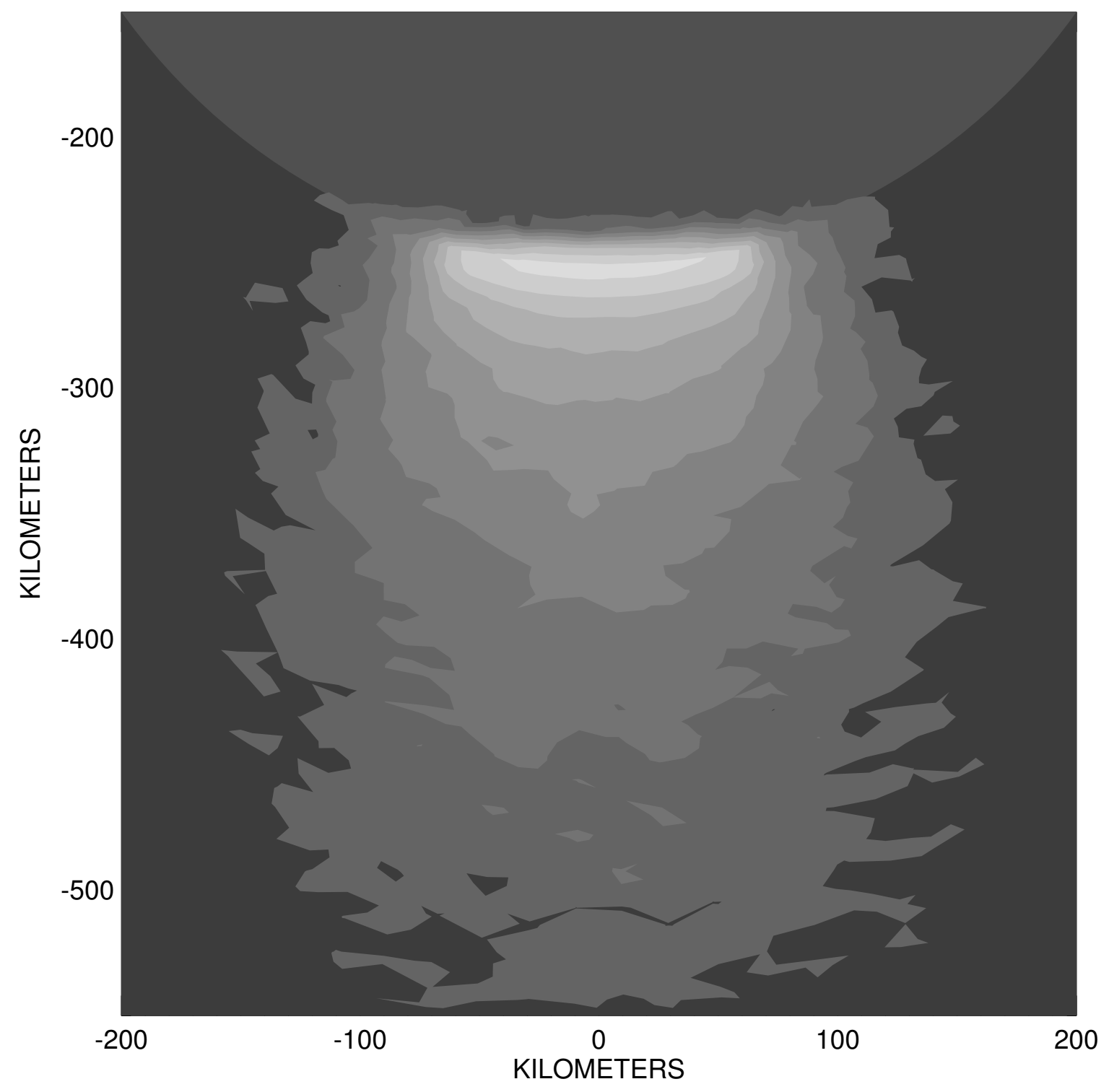




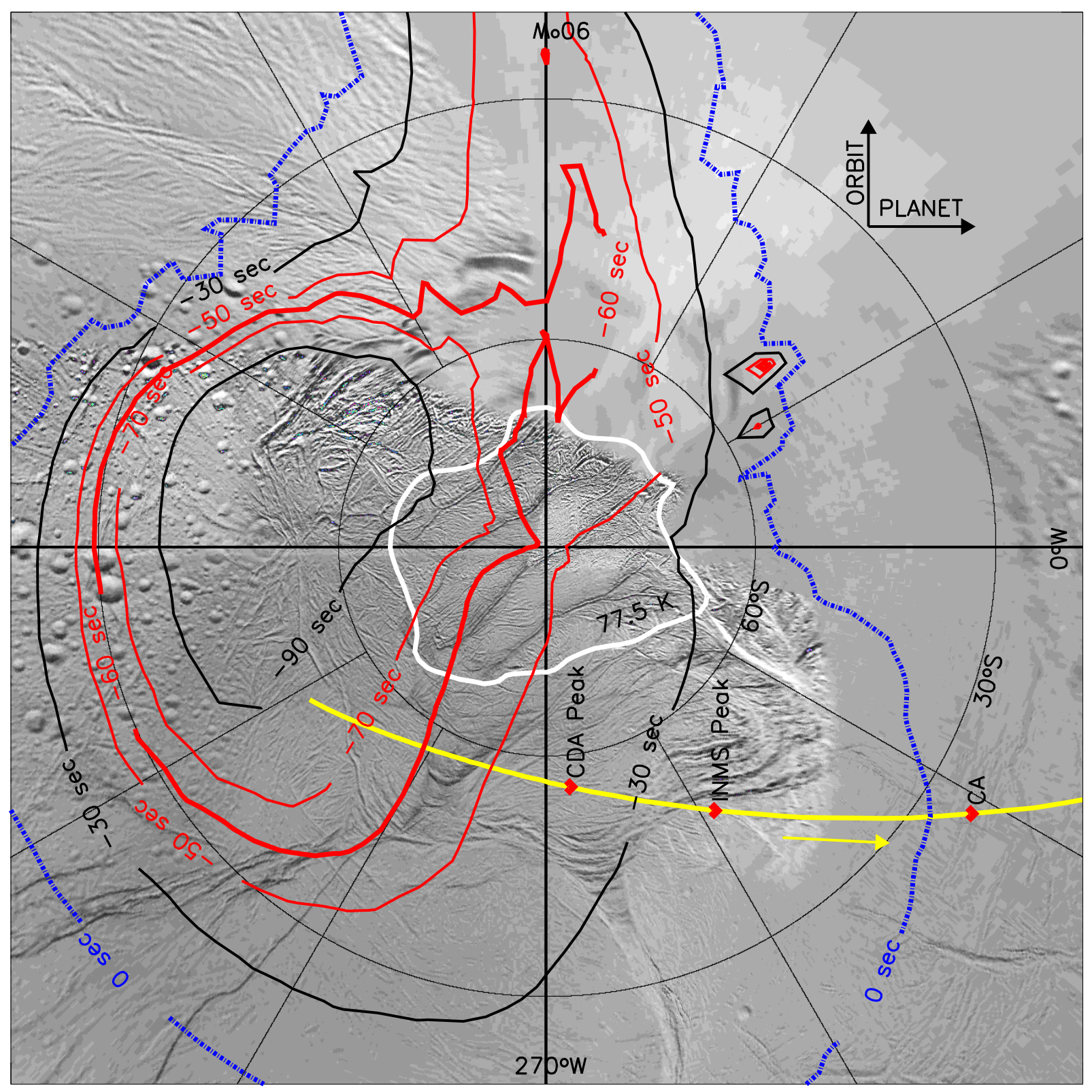




\title{
Supporting Online Material for
}

\section{Cassini Dust Measurements at Encleadus: Implications for Saturn's E Ring}

\author{
Frank Spahn*, Jürgen Schmidt, Nicole Albers, Marcel Hörning, \\ Martin Makuch, Martin Seiß, \\ Sascha Kempf, Ralf Srama, Valeri Dikarev, \\ Stefan Helfert, Georg Moragas-Klostermeyer, \\ Alexander V. Krivov, Miodrag Sremčević, \\ Anthony Tuzzolino, Thanasis Economou, Eberhard Grün \\ * to whom correspondence should be adressed, Email: \\ fspahn@agnld.uni-potsdam.de
}

File includes:

Materials and Methods

Figs. S1 to S7

Notes and References 


\section{Introduction}

In this appendix we provide additional material, methods and information which had been necessary to perform the analyses presented in the main paper. This concerns mainly information about the impactor ejecta process, the spatial ejecta-distribution and the dust dynamics in order to obtain the dust configuration around the satellite Enceladus, which is material mentioned only marginally in the main paper.

\section{The Impactor-Ejecta Mechanism}

Micrometeroid impacts in the solar system are energetic enough to abundantly lift debris and dust particles from the moon's surfaces. A model of an impactgenerated steady-state dust cloud around an atmosphereless planetary satellite has been developed $[1,2]$. In this model, dust grains are ejected within a cone of an opening angle $\Delta \alpha$. Their initial velocity is determined by the distribution $f_{v}$ of ejection speed $v$

$$
f_{v}=\frac{1}{v_{0}}\left(\frac{v}{v_{0}}\right)^{-q} \Theta\left[v-v_{0}\right]
$$

and ejection angle $\alpha$, filling the cone uniformly, as shown in Fig. S1. The Heaviside function is labeled by $\Theta\left[v-v_{0}\right]$ restricting the range of velocities to the interval $\left(v_{0}, \infty\right)$. The slope of the power law has been chosen as $q \in(2,3)$ depending on whether the surface is covered by regolith or consists of solid ice.

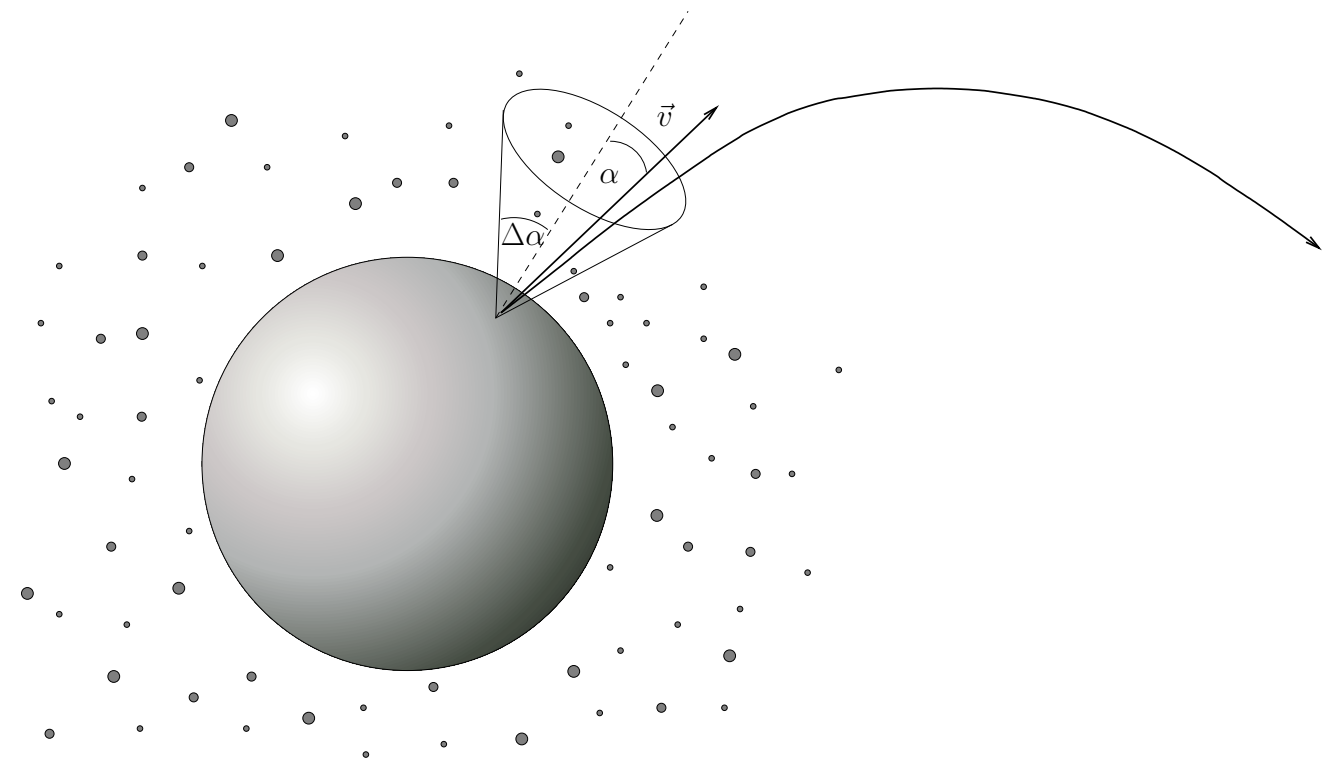

Figure S1: Geometry of the ejection process. 
Within the Hill sphere of radius $r_{h}$, which denotes Enceladus' range of gravitational influence,

$$
r_{h}=a_{E} \sqrt[3]{\frac{M_{E}}{3\left(M_{E}+M_{S}\right)}}
$$

all perturbing forces besides the moon's gravity are negligibly small and were neglected $[1,2]$ to describe the dust clouds around atmosphereless celestial bodies. The masses of Saturn, Enceladus and the semi-major axis of the latter are denoted by $M_{S}, M_{E}$ and $a_{E}$, respectively. The comparison of the analytical model to data obtained with the Galileo dust detector $[3,4]$ proved the existence of these dust clouds for the Galilean moons.

Although the dynamics of a dust grain in the vicinity of Enceladus is almost independent of the mass $m$ (mass-dependent perturbations are negligibly small inside the Hill sphere), the size distribution remains an important characteristics of the dust production process. For the impactor ejecta mechanism a power law has been derived from field experiments and theoretical studies (for details see Krivov et al. [1])

$$
N^{+}\left(>R_{p}\right) \propto R_{p}^{-12 / 5}
$$

for the number of particles larger than a certain radius $R_{p}$.

The size distribution can be derived from HRD data yielding a power law $N^{+} \approx R_{p}^{-b}[b \in(2.5,3.0)]$ representing a dependency often observed for geophysical processes.

\section{Equations of motion and perturbation forces}

After the particles have been created and lifted off from the surface of their parent body they are governed by forces acting in Saturn's environment.

In the direct vicinity of a satellite the dynamics of a particle is affected mainly by the gravity of the satellite and to smaller extent by the planet described by the three-body problem

$$
\ddot{\mathbf{r}}=G \nabla\left(\frac{M_{S}}{\left|\mathbf{r}-\mathbf{r}_{\mathbf{S}}\right|}+\frac{M_{E}}{\left|\mathbf{r}-\mathbf{r}_{\mathbf{E}}\right|}\right)
$$

where $G$ is the gravitational constant. The positions of the dust particle, and Saturn's and Enceladus' center are labeled by $\mathbf{r}, \mathbf{r}_{\mathbf{S}}$, and $\mathbf{r}_{\mathbf{E}}$. Trajectories were integrated using Eqs. (4) until the particles leave Enceladus' vicinity (we define as the region inside $5 r_{h}$ ) or until they fall back on the moon's surface. Analytical expressions for the flux of dust originating at Enceladus can be derived if Saturn's tides, the first term in Eqs. (4), were neglected. This is an useful description for the region inside Enceladus' Hill sphere discussed in figure $\mathrm{S} 2$. In order to obtain the dust impact rates expected at the Cassini-HRD in a 
larger domain around the satellite Eqs. (4) must be solved numerically. These results are discussed below in the context with figure S3.

For the simulation of the E ring background, which is an important contribution to the HRD signal besides the dust coming directly from Enceladus, non-gravitational perturbations have to be taken into account in order to address the long-term dynamics of an $\mathrm{E}$ ring grain from its creation until its annihilation at the certain sinks. The complete equations of motion read $[6,8]$

$$
\begin{aligned}
\ddot{\mathbf{r}}= & G \nabla\left(\frac{M_{S}}{\left|\mathbf{r}-\mathbf{r}_{\mathbf{S}}\right|}-\frac{M_{S} R_{S}^{2}}{\left|\mathbf{r}-\mathbf{r}_{\mathbf{S}}\right|^{3}} \mathcal{J}_{2} \mathcal{P}_{2}(\cos \theta)+\frac{M_{E}}{\left|\mathbf{r}-\mathbf{r}_{\mathbf{E}}\right|}\right) \\
& +\frac{Q}{m}(\mathbf{E}+\dot{\mathbf{r}} \times \mathbf{B}) \\
& -\frac{Q_{P R}}{m} \frac{\pi R_{p}^{2}}{c} q_{\odot}\left(\frac{R_{\oplus}}{R}\right)^{2} \mathbf{e}_{\odot} \\
& -\pi n_{I} m_{I} R_{p}^{2} u^{2} \mathbf{e}_{\mathbf{u}}
\end{aligned}
$$

where the acceleration terms signify in the order or their appearance the gravity of oblate Saturn and Enceladus, the Lorentz force, solar radiation pressure, and plasma drag. The angle $\theta$ denotes the angular distance of the particle's position $\left(\mathbf{r}-\mathbf{r}_{\mathbf{S}}\right)$ from the north pole $\mathbf{e}_{z}$. The second harmonic $J_{2} \approx 1.67 \cdot 10^{-2}$ measures the oblateness of Saturn responsible for the deviations of its potential from spherical symmetry. The opposing dynamical effects of Saturn's oblateness and the Lorentz force select the 1 micron particles to make up mainly the E ring, its shape and extent [7]. Here we are interested in larger particles $\left(R_{p}>2 \mu \mathrm{m}\right)$ registered by the HRD which stay closer to their sources.

The induced electric $\mathbf{E}$ and the magnetic field $\mathbf{B}$ are given by

$$
\begin{aligned}
& \mathbf{E}=\left\{\left(\mathbf{r}-\mathbf{r}_{\mathbf{S}}\right) \times \Omega_{S}\right\} \times \mathbf{B} \\
& \mathbf{B}=\frac{\mathbf{B}_{\mathbf{0}}}{\left|\mathbf{r}-\mathbf{r}_{\mathbf{S}}\right|^{3}}-\frac{3\left(\mathbf{r}-\mathbf{r}_{\mathbf{S}}\right)\left[\mathbf{B} \cdot\left(\mathbf{r}-\mathbf{r}_{\mathbf{S}}\right)\right]}{\left|\mathbf{r}-\mathbf{r}_{\mathbf{S}}\right|^{5}}
\end{aligned}
$$

where $\boldsymbol{\Omega}_{S}=1.64 \cdot 10^{-4} \mathrm{sec}^{-1}$ and $\mathbf{B}_{\mathbf{0}}=B_{0} R_{S}^{3} \mathbf{e}_{z}$ with $B_{0}=-2 \cdot 10^{-5} \mathrm{Vs} \mathrm{m}^{-2}$ are Saturn's rotation period and dipole moment, respectively.

The speed of light, radiation pressure coefficient, and the solar constant are denoted by $c, Q_{P R}$, and $q_{\odot}=1.37 \mathrm{~kW} \mathrm{~m}^{-2}$, respectively. The distances Sun-particle and Sun-Earth are given by $R=\left|\mathbf{r}-\mathbf{r}_{\odot}\right|$ and $R_{\oplus}$. The vector $\mathbf{e}_{\odot}$ points from the particle to the Sun, i.e. the direct radiation pressure force acts in anti-Sun direction.

The direct plasma drag is essentially determined by the density $n_{I}$ and mass $m_{I}$ of heavy ions and the relative velocity $\mathbf{u}=\dot{\mathbf{r}}-\Omega_{S} \times\left(\mathbf{r}-\mathbf{r}_{\mathbf{S}}\right)$ between the dust-grain and the plasma. For the E ring of Saturn the indirect Coulomb drag component is negligibly small and the grain velocities are supersonic, $|\mathbf{u}| / v_{t h} \gg 1\left(v_{t h}-\right.$ thermal speed of heavy ions). Thus, we can apply the simple formulation of the plasma drag [8] in Eq. (5). 
Equations (5) have been solved numerically for grains of certain sizes $(1,2,3$, $4, \ldots 10 \mu \mathrm{m})$ just emitted from the moon's surface at rates corresponding to both creation processes. This gives $\approx 10^{13} \mathrm{~s}^{-1}$ particles escaping the gravity of Enceladus. Their trajectories were followed until the particles hit Enceladus, other E ring moons or they reach the main rings. The phase space coordinates of each particle were stored in equidistant time-intervals. In this way a stationary phase-space density $n(\mathbf{r}, \dot{\mathbf{r}})$ of the population can be constructed from which the impact rate onto the Cassini HRD of larger particles $\left(R_{p}>2 \mu \mathrm{m}\right)$ in the vicinity of Enceladus' orbit can be calculated. Using this, contributions of freshly ejected particles at Enceladus originating from the impactor-ejecta process and the south-pole source have been combined with the E ring background to give the total model rate (see Fig. 1, main paper) expected at the Cassini HRD.

\section{Localized ejecta start positions}

In the main paper the comparison between the expected dust impact rates with the Cassini HRD data is presented and the moment of maximum-rate is outed as a decisive quantity. In order to relate this moment in time with a certain dust source region at Enceladus' surface various simulations based upon the two-body as well as the three body equations of motion have been performed. Figs. S2 and S3 depict the time offset of the moment of the rate-maximum from the closest approach for various ejection points on Enceladus' surface by different colors. The actual Cassini flyby trajectory is projected onto the surface (blue line) and the point of closest approach is especially marked (red point). The two different presentations base upon the two-body problem (first term in Eq. (4) neglected, Figure S2) and the three-body problem (4) (Figure S3).

According to the results of the flyby E11 the regions from which the dust grains most likely originate from are shown in red, corresponding to the observed time offset of - 1 minute of the maximum peak rate to that of the closest approach. In addition the brightness measures the intensity of the dust flux expected at the dust-detector where dark and bright signify small and large fluxes at the HRD corresponding to a low and a high efficiency of the source region, respectively.

From the Figures S2 and S3 one may deduce that both approaches, the two-body as well as the three-body description, lead to comparable results. This means that the analytical solution in terms of the two-body problem provides reasonably good results for flyby sections well inside the Hill-sphere of gravitational influence, which applies to Cassini's position when the maximum rate occurred (red areas in the plot). 


\section{Influence of start positions and velocities}

In order to study the source region we performed simulations where dust grains were ejected according to a 2D Gaussian distribution of start positions from the satellite's surface with the mean at the south pole. Then, the size of the source region is characterized by the standard deviation $\sigma$. Using a $2 \mathrm{D}$ Gaussian or a uniform distribution - as in the main paper - yields nearly the same impact rates along the flyby trajectory (Fig. S4). The influences of width $\sigma$, cone opening angle $\Delta \alpha$, and the maximum ejecta speed $v_{\max }$ on the impact rate and offset times are illustrated in Figs. S5, S6, and S7.

\section{References}

[1] A. Krivov, M. Sremčević, F. Spahn, Planet. Space Sci., 51, 251 (2003).

[2] M. Sremčević, A. Krivov, F. Spahn, Planet. Space Sci., 51, 455 (2003).

[3] H. Krüger, A. Krivov, M. Sremčević, E. Grün, Icarus, 164, 170 (2003).

[4] M. Sremčević, A. Krivov, H. Krüger, F. Spahn, Planet. Space Sci., 53, 625 (2005).

[5] D. Hamilton, J. Burns, Science, 264, 550 (1994).

[6] F. Spahn, K.-U. Thiessenhusen, J. E. Colwell, R. Srama, E. Grün, J. Geophys. Res., 104, 24111 (1999).

[7] M. Horányi, J. A. Burns, D. P. Hamilton, Icarus 97, 248 (1992).

[8] V. V. Dikarev, Astron. Astrophys. 346, 1011 (1999). 

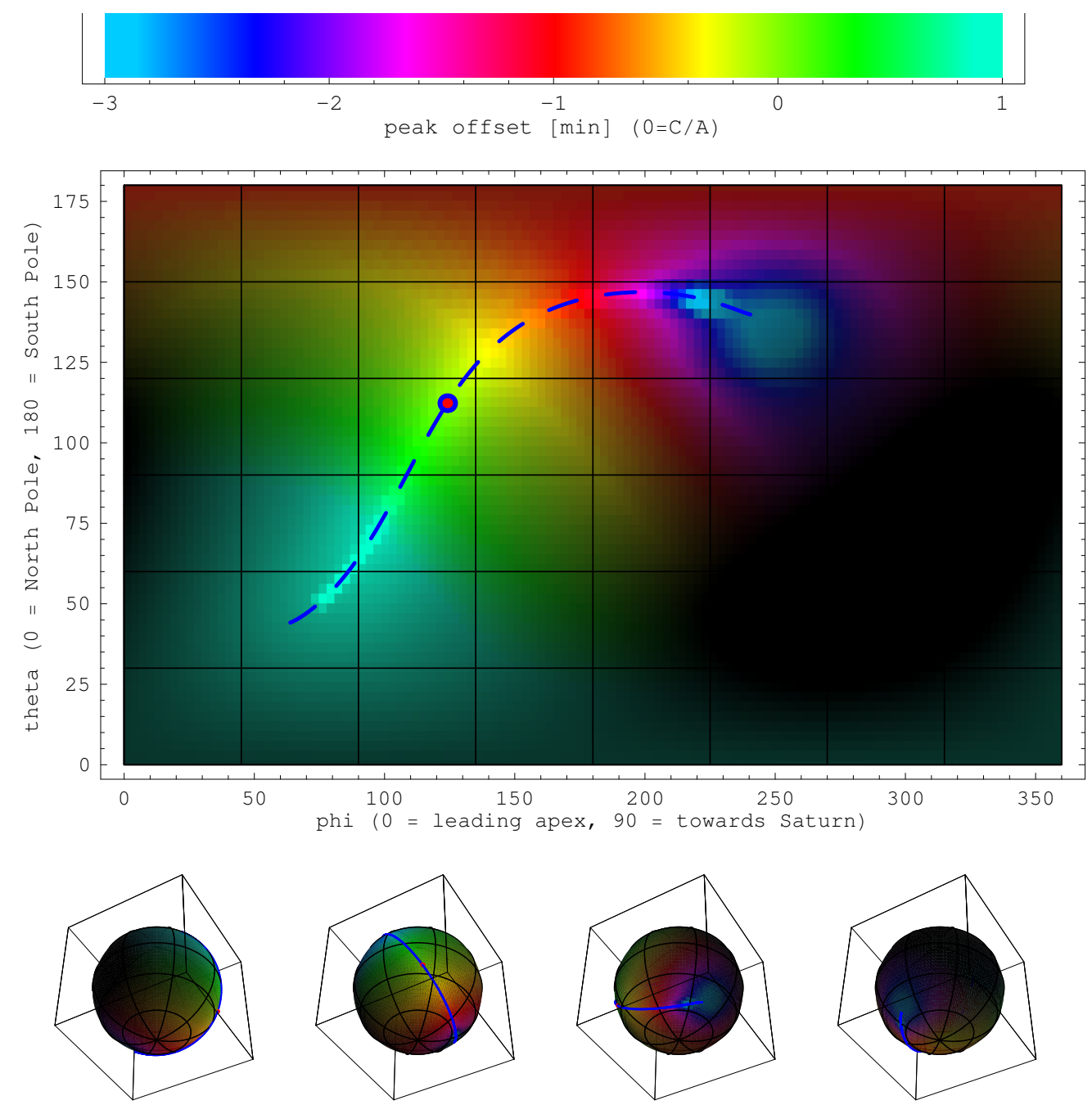

Figure S2: Analytical solution in terms of the two-body problem similar to models of $[1,2]$, but with dust particles produced from a surface point instead of an extended area. A point source on the surface of the moon in spherical coordinates at $(\theta, \phi)$ has been chosen with a non-zero cone opening angle in the interval $\left[0^{\circ}, 45^{\circ}\right]$ and a mean $\langle\Psi\rangle \approx 9^{\circ}$ strongly favoring normal ejecta, and the velocities are assumed to obey a differential power law distribution with index -3 . With these assumptions, the time offset with respect to the closest approach is represented by colors (color scale above). Brighter and darker regions refer to stronger and weaker impact signals and thus give information about the relative efficency of a source at given location. Clearly the bright regions are expected close to the ground-track of the Cassini trajectory, indicated by the blue dashed line where the red dot is the moment of closest approach. Dark regions are located diametrically opposite from the trajectory track. The closest approach is just where the offset time is zero (green area). The lower panels provide a 3D illustration of the color coded time offset with a view onto the south pole of Enceladus (from left to right: rotations of $90^{\circ}$ from leading apex to the side opposite to Saturn). 

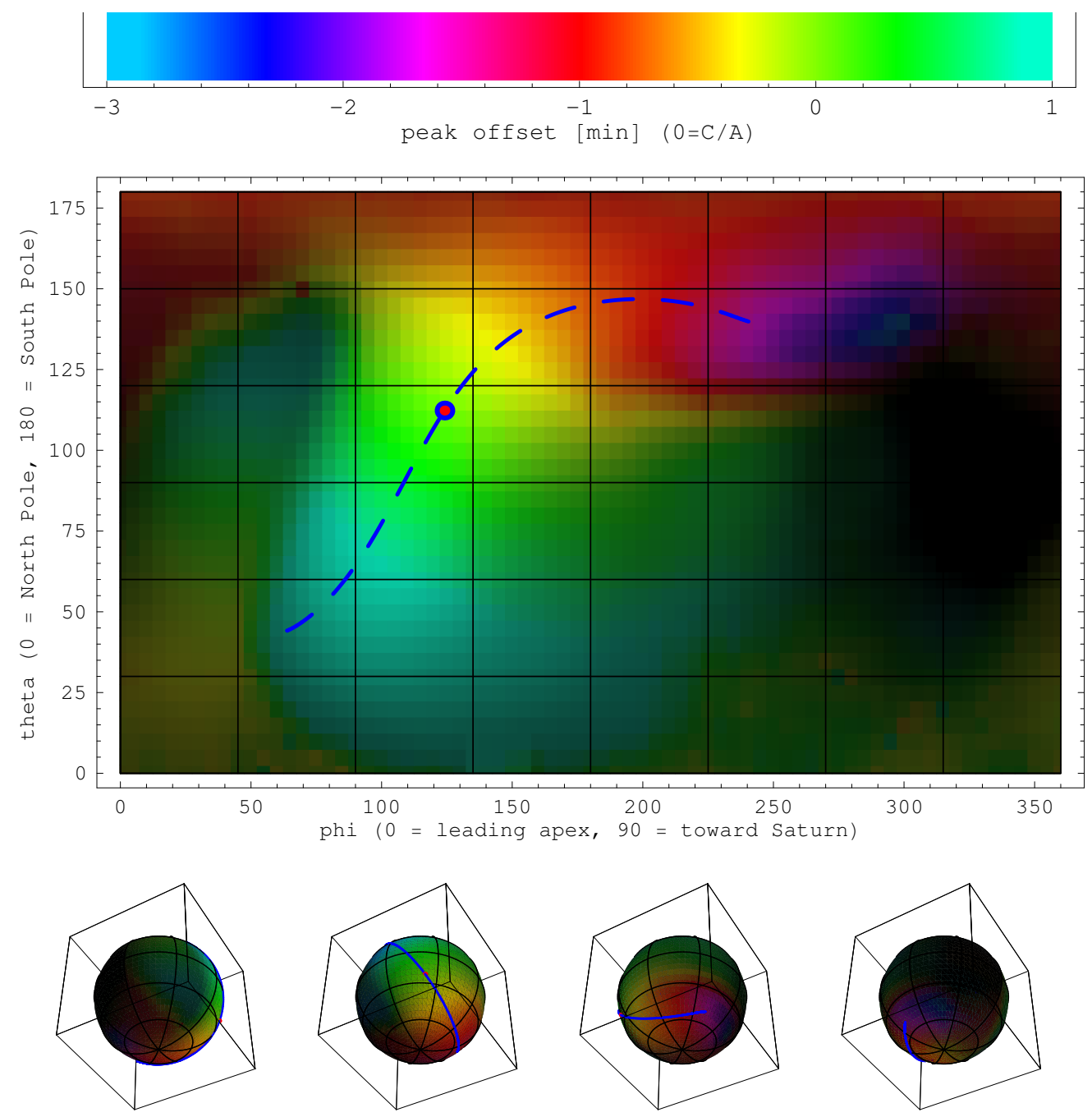

Figure S3: Numerical solution of the full three-body problem [Eqs. (4)] using the same notation and illustration as in Fig. S2. The ejected dust grains originate from a small surface area of a (semi-) angular width of $\xi=15^{\circ}$ with an ejecta cone opening angle of $25^{\circ}$. Within this area the start positions are distributed isotropically and a differential power law velocity distribution with index -2 was used. The overall picture is similar to the analytic solution (Fig. S2). The main differences arise since the analytic solution is considering the two-body problem and is thus applicable within the Hill sphere (corresponding to just \pm 90 seconds around closest approach). 


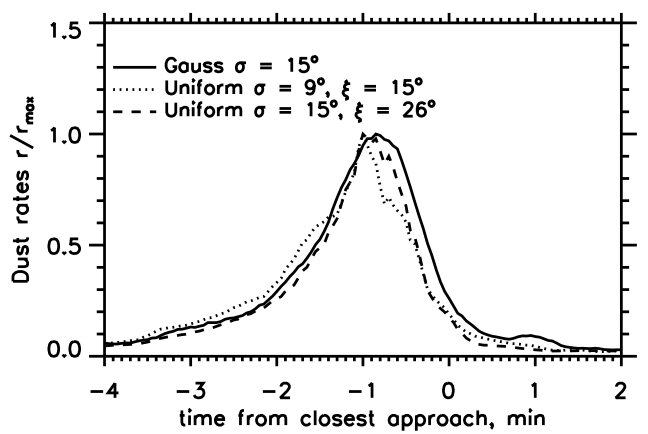

Figure S4: Comparison between a 2D Gaussian and a uniform distribution of the ejecta starting positions on the surface. In the latter case particles were ejected from a source region of (semi-) angular width of $\xi$. Using the same standard deviation $\left(\sigma=\sqrt{\left\langle\xi^{2}\right\rangle-\langle\xi\rangle^{2}}\right)$ yields similar impact rates along the flyby trajectory.
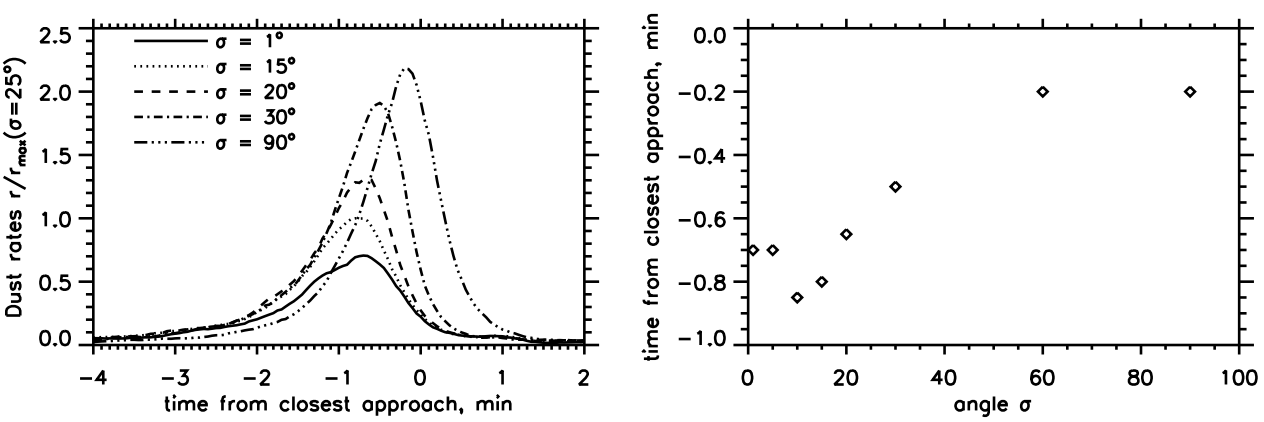

Figure S5: Dependence of impact rate and offset time on the standard deviation $\sigma$ of the 2D Gaussian distribution. It has been varied for angles of $1^{\circ}$, $5^{\circ}, 10^{\circ}, 15^{\circ}, 20^{\circ}, 30^{\circ}, 60^{\circ}$, and $90^{\circ}$. The left panel shows the dust rate along the flyby trajectory for different width angles $\sigma$. The rates are normalized with respect to the maximum rate of the model presented in the main paper ( $\sigma=15^{\circ}, \Delta \alpha_{\max }=25^{\circ}$, differential power law for the velocity dependence in the range of 0.5 until 2.0 escape velocities with a power law index of -2 ). The rate maxima increase with growing $\sigma$. The right panel shows the offset time for different angles $\sigma$. The largest modulus of the offset time is obtained for a width of about $10^{\circ}$. For larger widths the offset time approaches zero. This can be understood by considering that large $\sigma$ correspond to the isotropic case. 

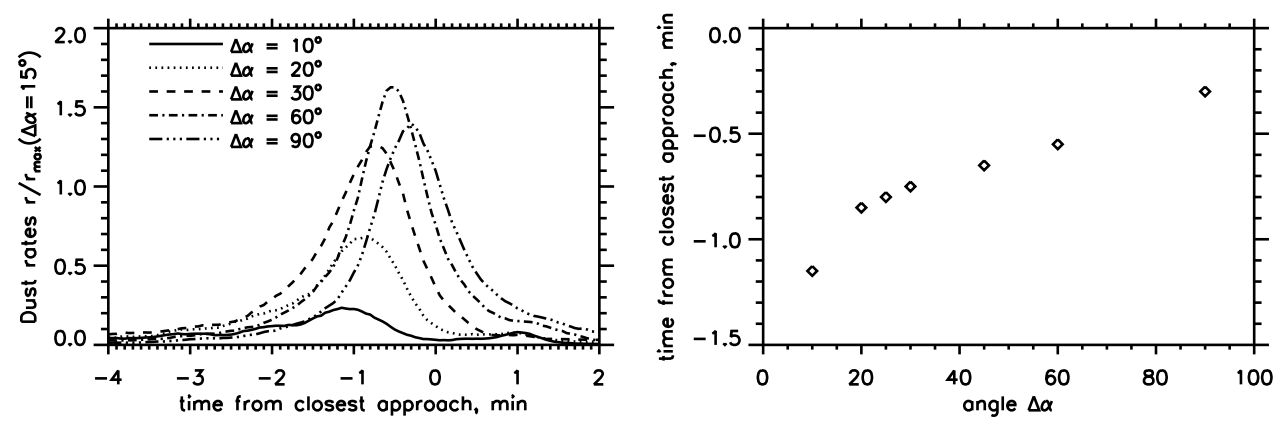

Figure S6: Dependence of impact rate and offset time on the maximum opening-angle of the ejecta cone. The ejecta cone angle $\Delta \alpha$ has been varied as $10^{\circ}, 20^{\circ}, 25^{\circ}, 30^{\circ}, 45^{\circ}, 60^{\circ}$ and $90^{\circ}$. The left panel shows the respective dust rate along the flyby trajectory. The rates are normalized as in Fig. S5. The maximum rate can be found for $\Delta \alpha \approx 60^{\circ}$ being an optimum for this trajectory. A wider ejection cone results in an decreased offset time (right panel) and larger rate maxima. According to these tests the value $\Delta \alpha=30^{\circ}$ yields the best agreement with the observed HRD-rate.
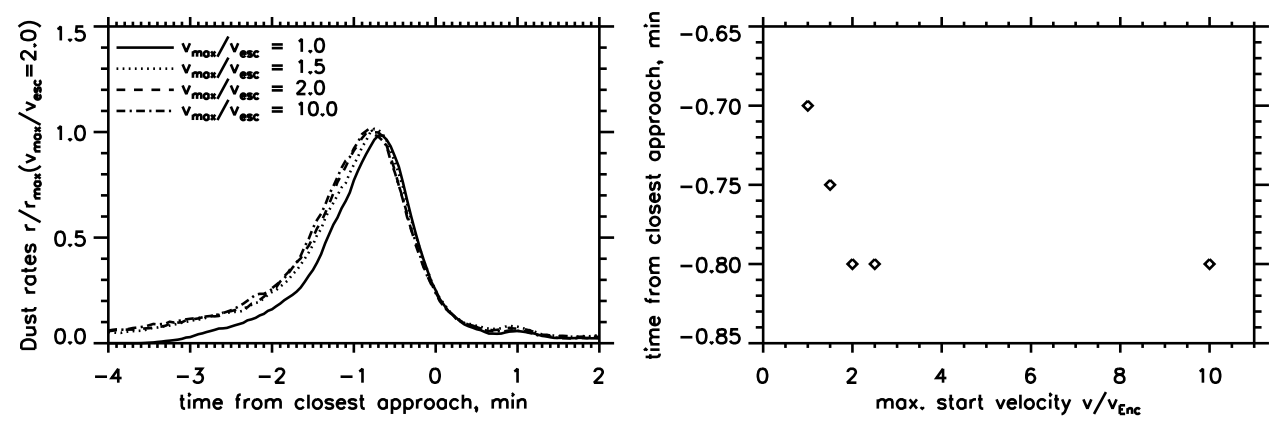

Figure S7: Dependence of impact rate and offset time on the maximum ejecta velocity. Values of 1.0, 1.5, 2.0, 2.5 and 10.0 escape velocities have been used for the maximum of the velocity distribution $v_{\max }$. The left panel shows the dust rate along the flyby trajectory for different maximum velocities. The rates are normalized as in Fig. S5. The right panel shows the offset time which is decreasing for $v_{\max }<2.0$ but leveling off for $v_{\max }>2.0$. 